\title{
Desinstitucionalización del sistema de partidos en México: volatilidad, fragmentación y número efectivo de partidos
}

\author{
Deinstitutionalization of the party system in Mexico: volatility, \\ fragmentation and effective number of parties
}

\section{José Manuel Luque Rojas}

\section{Resumen}

El presente trabajo analiza el proceso de desinstitucionalización del sistema de partidos en México observable a partir la constante desafección de la ciudadanía respecto de los tres principales partidos políticos de México, que construyeron el proceso de democratización del anterior régimen autoritario de tipo hegemónico, en el actual régimen democratizado con profundas huellas de desencanto. Se utilizan variables e indicadores politológicos como la volatilidad, la fragmentación electoral y el número efectivo de partidos y se intenta ofrecer explicaciones de las causas que han provocado la caída en los niveles de apoyo electoral a los tres partidos principales a favor de otras opciones políticas.

\section{Palabras clave}

Desinstitucionalizacion; Sistema de Partidos; Volatilidad; Fragmentación y Numero Efectivo de Partidos; México.

\begin{abstract}
This paper analyzes the process of deinstitutionalization of the party system in Mexico observable from the constant disaffection of citizenship with respect to the three main political parties in Mexico, which built the process of democratization of the previous hegemonic type of authoritarian regime in the current Democratized regime with deep traces of disenchantment. Political variables and indicators such as volatility, electoral fragmentation and the effective number of parties are used and attempts are made to explain the causes that have caused the fall in levels of electoral support to the three main parties in favor of other political options.
\end{abstract}

\section{Keywords}

Deinstitutionalization; Party System; Volatility; Fragmentation and Effective Number of Parties; Mexico. 


\section{Introducción}

México experimentó un largo proceso de transición a la democracia, inició formalmente con la reforma política de 1977 y concluyó el primero de diciembre del ańo 2000 con la toma de protesta del panista Vicente Fox Quezada el primer presidente de la república emanado de un partido de oposición en 71 años. La reforma de 1977 abrió el régimen político a la participación (muy restringida) de todas las oposiciones de izquierda y de derecha, en los siguientes 15 ańos nació y se institucionalizó un sistema de partidos de tres partidos principales: PRI, PAN y PCM-PRD y varios periféricos, este sistema tripartidista en los siguientes 10 ańos entra en un proceso de desgaste y amplio rechazo de la ciudadanía en todo el país.

El presente ensayo tiene como objetivo analizar el fenómeno de la desinstitucionalización del sistema de partidos en México, que ha tenido una expresión especialmente fuerte en las recientes elecciones federales de 2015, en el que la preferencia electoral hacia los partidos tradicionales, medida en sus porcentajes de votación descendió a mínimos históricos. En oposición a esto, la emergencia de nuevos partidos, de partidos antes marginales y candidatos independientes, creció electoralmente lo que se refleja con el aumento en los índices de volatilidad, fragmentación, el número efectivo de partidos y voto escindido, desviado o realineado.

La desinstitucionalización del sistema de partidos en México entendida como el desalineamiento electoral de los ciudadanos y la merma de la identidad partidaria tradicional que mantuvo estables los niveles de concentración electoral por los tres principales partidos políticos en los procesos electorales de las décadas de 1990 y 2000 y observable en la evolución de los índices referidos en el párrafo anterior, la disminución del partidismo y el crecimiento de los independientes y del voto escindido, es un claro indicador de desafección hacia los partidos tradicionales y el debilitamiento del sistema de partidos configurado en el proceso de democratización del régimen político mexicano.

El trabajo sostiene la hipótesis de que está en marcha un proceso de desinstitucionalización del sistema de partidos en México, que se expresa a través del aumento gradual de los índices de volatilidad (PEDERSEN, 1979) fragmentación electoral (SARTORI, 1987; OÑATE y OCAÑA, 1999); voto escindido (COLOMER, 2001; SANZ CAZORLA, 2005; JACOBSON, 1990; MONTERO, 1988; PALLARÉS y KEATING, 2006; DE VRIES y TERRANCE, 1972; FIORINA, 1992) y el número efectivo de partidos (LAAKSO y TAAGEPERA, 1979). Este fenómeno a su vez es expresión de desencanto del desempeño 
gubernamental y legislativo de los tres principales partidos políticos: el Partido Revolucionario Institucional (PRI), el Partido Acción Nacional (PAN) y el Partido de la Revolución Democrática (PRD), en los sucesivos ejercicios de gobierno federal en el caso de los dos primeros y de gobiernos locales y del DF en el caso del PRD, y en el poder legislativo de los tres institutos políticos.

Los datos que se analizan son los resultados de las elecciones federales de diputados de 1997, 2000, 2001, 2003, 2006, 2009, 2012 y 2015, tomados de la página del Instituto Nacional Electoral (INE), mismos que serán trabajados bajo tres fórmulas distintas para obtener los índices de volatilidad y fragmentación electoral, el del número efectivo de partidos y el voto escindido. Los componentes matemáticos de las fórmulas y el sustento teórico de estos se expondrán someramente en cada apartado.

El trabajo se expone en seis apartados: en el primero se exponen los antecedentes de la conformación y consolidación del sistema de partidos en México; en el segundo se analiza la evolución del índice de concentración y fragmentación electoral entre 1997-2015; en el tercero se exponen los datos del índice de volatilidad electoral; en el cuarto, se estudia la evolución del indice del número efectivo de partidos en México en el periodo de estudio, en el quinto, se analizan algunas causas probables de la desafección ciudadana hacia los partidos tradicionales y la crisis de desinstitucionalización, y en el sexto y último está dedicado a hacer un análisis del conjunto de las variables históricas y politológicas a manera de conclusiones.

\section{Antecedentes del sistema de partidos en México}

Los antecedentes del sistema de partidos los podemos dividir en dos grandes periodos, el que va de 1929 a 1976 y el que inicia en 1977 a 2015. En el primero, dominado totalmente por el nacimiento del Partido Nacional Revolucionario (PNR) en 1929 y sus posteriores transformaciones en Partido de la Revolución Mexicana (PRM) en 1938, y Partido Revolucionario Institucional (PRI) en 1946. Estos partidos son herederos del régimen revolucionario e institucionalizaron el ejercicio del poder a partir de la legitimidad que les brindó en su origen el movimiento armado. Más allá de las oportunidades que la coyuntura internacional de la posguerra brindó al PRI para gestionar la modernización industrial y el crecimiento económico, este partido centralizó el poder, los recursos y los votos, configurando un sistema de partido hegemónico en torno al cual giró la vida política del país entre 1940 a 2000 . 
Al mismo tiempo del afianzamiento del régimen de partido hegemónico, nacen dos partidos, que en esencia fueron escisiones del proyecto revolucionario en las décadas de 1930 y 1940. El Partido Acción Nacional (PAN) en 1939 y el Partido Popular Socialista (PPS) en 1948. En 1954, una nueva escisión de antiguos militares revolucionarios formaron el Partido Auténtico de la Revolución Mexicana (PARM), estos tres partidos con reconocimiento y registro legal, más el PRI como partido principal, configuraron el sistema de partido hegemónico en México, hasta la reforma de 1976-77. PAN, PPS y PARM acompañaron al PRI en los rituales electorales entre 1946 hasta 1976, los resultados no vale la pena analizarlos, el monopolio de votos y representación era exclusiva del partido hegemónico: el PRI, una rápida mirada a los resultados de las elecciones presidenciales en el periodo lo confirman, en las elecciones de 1940 obtuvo oficialmente 93,9\% de los votos, en 1946 el 77,9\%, en 1952 el $74,32 \%$, en 1958 el 89,81\%, en 1964 el 88,81\%, en 1970 el 84,79\%, en 1976 el $91,9 \%$ del total de votos y en este último, el $100 \%$ de los votos válidos porque no hubo opositores legales registrados.

Como puede verse, este fue un sistema de partidos en los que la competitividad no existió, acorde a la naturaleza de los regímenes hegemónicos, las reglas de la competencia no permitieron la disputa real de los cargos. En la práctica si existió la oposición electoral, en algunas elecciones quizá realmente competitiva: 1940, 1952 y 1958 al menos, pero la naturaleza autoritaria y las reglas del régimen impidieron se expresara en los resultados oficiales. Las denuncias de fraudes por parte de los candidatos opositores, Juan Andrew Almazán en 1940, del general Miguel Enríquez Guzmán en 1952 y de Luis Héctor Álvarez en 1958, respectivamente, son indicadores de esto (AGUILAR y MEYER, 1991).

La génesis del actual sistema de partidos en México la podemos encontrar en la reforma política de 1976-1977. Con aquella reforma liberalizadora inicia el periodo transicional en el país, y el cambio principal consistió en abrir el proceso para la formación y reconocimiento legal de nuevos partidos políticos, especialmente a la izquierda que había activado políticamente desde la década de 1920, y legalmente proscrito desde el afianzamiento del partido hegemónico en la década de 1940. La crisis política generada, en parte, por la crisis desatada por el fin del ciclo de crecimiento económico de la posguerra a finales de la década de 1960, en parte por los excesos autoritarios de los gobiernos de Gustavo Díaz Ordaz y Luis Echeverría Álvarez, que como respuesta a las manifestaciones, huelgas y marchas de campesinos, obreros, profesionistas y estudiantes, recurrieron a la represión brutal, sembrando el terror, lo que modificó el rostro del régimen hegemónico de ser agregador, eficiente y 
tolerante, a ser represor y corrupto. Los conflictos con la clase empresarial derivado del reproche de esta al gobierno de Luis Echeverría de provocar con sus acciones y cerrazón un clima de discordia y de guerrilla, a propósito del secuestro y asesinato de Eugenio Garza Sada, presidente del grupo financiero Monterrey, por parte de guerrilleros de la liga comunista 23 de septiembre, quebró la alianza tradicional de parte importante del sector empresarial con el gobierno, lo que elevó los niveles de ilegitimidad del régimen hegemónico. Las elecciones Presidenciales y legislativas de 1976, paradójicamente fueron ganadas por el PRI con el 100\% de los votos válidos, pues el ingreso masivo de empresarios en el Partido Acción Nacional, provocó fuertes disputas internas y no postularon candidato presidencial dejando al PRI solo, lo que agravó la crisis de legitimidad, sin oposición el régimen se desnudó hacia adentro y hacia fuera como un régimen totalmente autoritario.

La salida de la crisis era solo una, la liberalización, el gobierno de José López Portillo la asumió, y envió apenas tomó protesta la propuesta de reforma liberalizadora en diciembre de 1976, se aprobó ese mismo mes y al año siguiente se expidió la Ley Federal de Organizaciones Políticas y Procesos Electorales (LFOPPE) (MÉXICO, 1977) que flexibilizó los requisitos para el registro de nuevos partidos, creó la figura de registro condicionado, por la que se multiplicaron los partidos en poco tiempo, federalizó su reconocimiento y emplazó a través de la reforma al artículo 116 de la Constitución Federal para que las entidades hicieran sus propias reformas para los regímenes locales, antes de las elecciones locales siguientes en cada caso, también disminuyó el umbral de votación mínima para el reparto de diputados de "Representación Proporcional" (RP) y fijó en 300 el número de diputados electos por el principio de mayoría relativa en todo el país, y creó 100 diputados de RP.

La LFOPPE modificó, entre otras cosas, la reglamentación del registro, funcionamiento, derechos y prerrogativas de los partidos; así de acuerdo a esa nueva ley electoral se establecieron dos rutas para obtener el reconocimiento legal como partido: a) Registro definitivo, y b) Registro condicionado. Bajo esta última figura varias organizaciones accedieron al ámbito partidista legal.

La LFOPPE señaló, en su artículo 32, que para obtener el registro condicionado al resultado de las elecciones, el solicitante debería acreditar que contaba con una declaración de principios, programa de acción y estatutos; que representaba una corriente de opinión "expresión de la ideología política característica de alguna de las fuerzas sociales que componen la colectividad nacional"; y haber realizado un actividad política permanente durante los cuatros ańos anteriores a la 
solicitud de registro (demostrada mediante reuniones, congresos, asambleas u otros actos políticos o bien, haber funcionado como asociación política nacional, un año antes de la convocatoria). Por otro lado, estableció que el partido político con registro condicionado al resultado de las elecciones obtendría el registro definitivo si obtenía al menos e 11,5\% de los sufragios, del total, en alguna de las votaciones de la elección para la que se le otorgó el registro condicionado. Y añadió que el hecho de que un partido político no obtuviera el registro definitivo no tenía efecto en relación con los triunfos que sus candidatos hayan obtenido en las elecciones según el principio de mayoría relativa

En las elecciones legislativas federales de 1979, se puso a prueba la eficacia de la recién estrenada (LGOPPE) además de los partidos tradicionales PAN, PRI, PPS y PARM, obtuvieron el registro condicionado los partidos: Comunista Mexicano (PCM), Demócrata Mexicano (PDM) y Socialista de los Trabajadores (PST), según los resultados oficiales, los siete partidos alcanzaron el registro definitivo al superar el umbral de 1,5\%, y con ellos se inicia el nuevo sistema de partidos políticos en el México de inicios de la Transición.

Con esta experiencia exitosa, más partidos solicitaron su registro y en las elecciones de 1982, participaron 9 partidos con registro: PAN, PRI, PPS, PARM, PSUM (antes PCM), PDM, y el PST más los partidos: Revolucionario de los Trabajadores (PRT) y el Social Demócrata (PSD). Como resultado de las elecciones de 1982, el PARM y el PSD perdieron su registro como partidos políticos nacionales al no obtener el 1,5\% de la votación que estipulaba la ley. No obstante, en 1984, el PARM solicitó ante la Comisión Federal Electoral su registro (ahora bajo la figura de definitivo) como partido político nacional. La comisión analizó la solicitud y después de la revisión de la misma decidió conceder de nuevo el registro al PARM (FLORES ANDRADE y ANSELMO, 2005).

En 1985, en las elecciones legislativas federales participaron de nuevo 9 partidos políticos, PAN, PRI, PSUM, PARM, PPS, PDM, PST, PRT y Partido Mexicano de los Trabajadores (PMT) fundado por Heberto Castillo. En esta elección perdió el registro el PRT, quedando ocho partidos con reconocimiento legal. Vale observar que a pesar de la cantidad de partidos con registro legal, los porcentajes de votación del PRI se mantuvieron más o menos estables: 69,71\% en 1979, 69,27\% en 1982 y 64,85\% en 1985 (FLORES ANDRADE y ANSELMO, 2005).

Con la fusión entre el PMT y el PST conformando el Partido del Frente Cardenista de Reconstrucción Nacional (PFCRN), y el Partido Mexicano Socialista (PMS) que sustituyó el registro del (PSUM), ocho partidos se presentaron a las 
elecciones de 1988 y cinco candidaturas: Cuauhtémoc Cárdenas encabezando al PARM, el PPS, el PFCRN y al PMS; Carlos Salinas de Gortari al PRI; Manuel de Jesús Clouthier del Rincón al PAN, Gumersindo Magaña al PDM y Rosario Ibarra de Piedra al PRT, esta elección marcó un parteaguas en la historia de la democratización, los resultados oficiales le dieron el triunfo al PRI, con denuncias de fraude, movimientos pos-electorales y abundantes evidencias de alteración de los resultados. El PRI descendió drásticamente en sus porcentajes, al registrar solo el $51,11 \%$ de la votación, pero con una oposición fragmentada que le dio amplio margen de victoria oficial sobre el FDN que encabezó Cuauhtémoc Cárdenas, a quien se le reconoció el 31\% de la votación Presidencial (FLORES ANDRADE y ALSELMO, 2005).

Las reformas electorales de 1986 habían ampliado a 200 el número de diputados de RP y estabilizado en 300 los de MR, por lo que el número de legisladores de la oposición al PRI en el Congreso se disparó al 48\% del mismo. Las reformas liberalizadoras tenían frutos visibles, la crisis económica de los últimos 15 años y la manipulación de las elecciones profundizaron el desprestigio del PRI y el gobierno, que los obligaron a escalar en la ruta reformista. En 1989 se hacen nuevos cambios en materia político electoral y se crea el Instituto Federal Electoral (IFE), y se introducen cambios regresivos en materia de candidaturas comunes, y alianzas electorales, la experiencia del FDN de 1988, les llevó a los priistas a cambiar las leyes electorales para prohibir los frentes, y aceptar solo alianzas o coaliciones con un solo logotipo, como si fuera un solo partido en las boletas electorales.

Las elecciones de 1991 y 1994, fueron organizadas por el IFE con fuerte control del gobierno y el PRI, bajo condiciones evidentes de inequidad en las elecciones, con excesiva presencia en los medios de este partido y restricciones legales y políticas fuertes hacia la oposición, especialmente de la izquierda. La reforma electoral de 1996, fue el gran arreglo político que generó por primera vez las condiciones para la competencia democrática, en términos de equidad, un elevado financiamiento público hacia los partidos políticos y acceso a medios de comunicación, sin más restricciones legales, más que las empresas televisivas y de la radio impusieran de manera diferenciada a los candidatos del PRI y los de la oposición. Formalmente se prohibió la extracción de recursos públicos de los gobiernos de todos los niveles para beneficiar al PRI o a cualquier otro partido. Con estos cambios democratizadores se celebraron las elecciones federales intermedias de 1997 y de varias entidades incluido por primera vez la elección de Jefe de Gobierno 
del Distrito Federal. Los resultados reflejaron el nivel de pluralidad de la sociedad mexicana, el reparto de gubernaturas, alcaldías, y la cámara de diputados del Congreso de la Unión fue plural. PAN, PRI y PRD, alcanzaron porcentajes que definieron la ruta tripartidista del sistema de partidos. Los niveles de concentración/ fragmentación es un buen indicador de estos resultados y los sucesivos.

\section{Concentración y fragmentación electoral 1997-2015}

Este apartado tiene el propósito de analizar el nivel de concentraciónfragmentación electoral de los partidos políticos en México a partir de 1997, se analizan siete elecciones federales a través del índice de concentración electoral o parlamentaria, que es un indicador que permite valorar el grado en el que el poder político está concentrado o disperso. El índice de concentración (C) se aplica tanto en la arena electoral como en la parlamentaria y se calcula mediante la siguiente formula: $\mathrm{C}=(\%$ del voto $\mathrm{A})+(\%$ del voto $\mathrm{B})+(\%$ del voto $\mathrm{C})$, siendo $\mathrm{A}, \mathrm{B}$ y $\mathrm{C}$, los partidos más votados

Debo aclarar, que originalmente el índice está diseñado para calcular la concentración electoral en solo dos partidos, pero aquí la hipótesis gira en torno a la afirmación de que en México, existe un sistema plural con tres partidos principales que desde 1997, institucionalizaron un sistema de partidos plural moderado y lo que se pretende probar es que dichos partidos, han ido perdiendo poco a poco su condición de partidos centrales y consecuentemente la necesaria reclasificación del sistema, a partir de las tendencias de desinstitucionalización de este sistema en favor de otro, que aún no puede definirse. 
Gráfico 1 - Concentracion/fragmentación, tendencia 1997-2015 dip (RP)

120

100

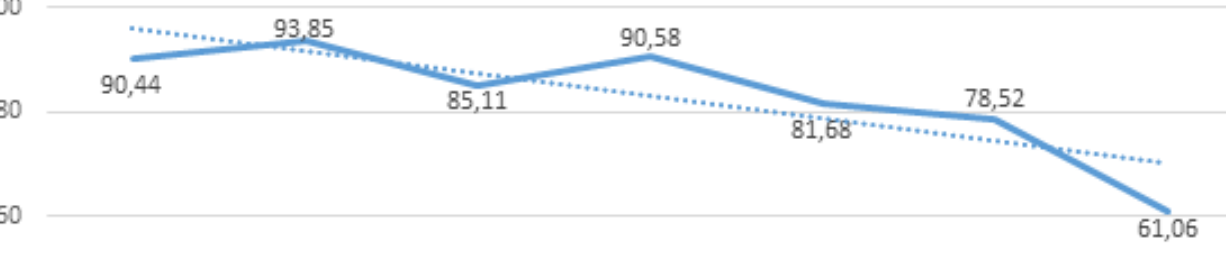

40

20

\begin{tabular}{|l|l|l|l|l|l|l|}
\hline PRI-PAN-PRD & PRI-PAN-PRD & PRI-PAN-PRD & PRI-PAN-PRD & PRI-PAN-PRD & PRI-PAN-PRD & PRI-PAN-PRD \\
\hline
\end{tabular} 1997 2000 2003 2006 2009 2012 2015

* resultados de elección de diputados por el principio de representación proporcional (RP). Fuente: Elaboración propia, datos del INE (atlas electoral).

Como puede verse, la tendencia marca un descenso en los niveles de concentración electoral de las tres principales fuerzas políticas del país. Entre 1997 y 2006, promediaron un índice de $90 \%$ de concentración, lo que significa que solo 10 electores de cada 100 votaron fuera de estas tres opciones electorales en las cuatro convocatorias del periodo. En cambio, las últimas tres elecciones, estos tres partidos solo promedian $73,75 \%$, es decir poco más de 26 electores en este periodo han optado por otros partidos políticos. Las causas de esta desafección hacia los tres principales partidos podemos encontrarlas quizá en la disminución del partidismo y el consecuente aumento del votante independiente que lo mismo puede votar por el partido con el que anteriormente mantenía una identificación o apego ideológico que por otras opciones de carácter más coyuntural o estratégico.

Hasta ahora, lo que se observa es una tendencia a la disminución de los niveles de concentración electoral entre los tres principales partidos, lo que puede razonablemente atribuirse a fallas en las estrategias de los partidos que merman su rendimiento, y que hace que el votante medio o estratégico que tradicionalmente distribuía o concentraba sus votos en alguna de estas tres opciones ahora lo haga por otras alternativas electorales de tipo partidista. Para despejar esta duda, analizaremos a 
partir del índice de volatilidad como ha repercutido la distribución de las pérdidas de votos en las últimas tres elecciones en cada uno de los partidos.

\section{Volatilidad electoral}

$\mathrm{La}$ volatilidad electoral es otros indicador que permite evaluar "la estructuración de la competencia partidista y su nivel de vinculación con el electorado" [informa sobre] "el nivel de alineamiento de los ciudadanos con los partidos, así como la estabilidad de las preferencias de los electores hacia un sistema de partidos determinado" (ALCÁNTARA, 2004, p. 38). Margarita Jiménez y Gabino Solano (2009) dicen al respecto:

Un punto a contemplar es el número de partidos que participan en cada proceso electoral, esto es, el que pierdan su registro unos partidos, o surjan nuevos y que participen en la contienda electoral, conlleva a incrementar el índice de volatilidad. El sistema de partidos se observa más consolidado cuanto más bajo sea el índice de volatilidad, indicando que, mientras más baja sea la volatilidad, mayor será la estabilización y consolidación del sistema de partidos (JIMÉNEZ y SOLANO, 2009, p. 43).

En el periodo 1997-2015 el índice de volatilidad agregado y por partidos se observa en las en el Gráfico 2: 
Gráfico 2 - Indice de volatilidad por partido y agregado 1997-2015

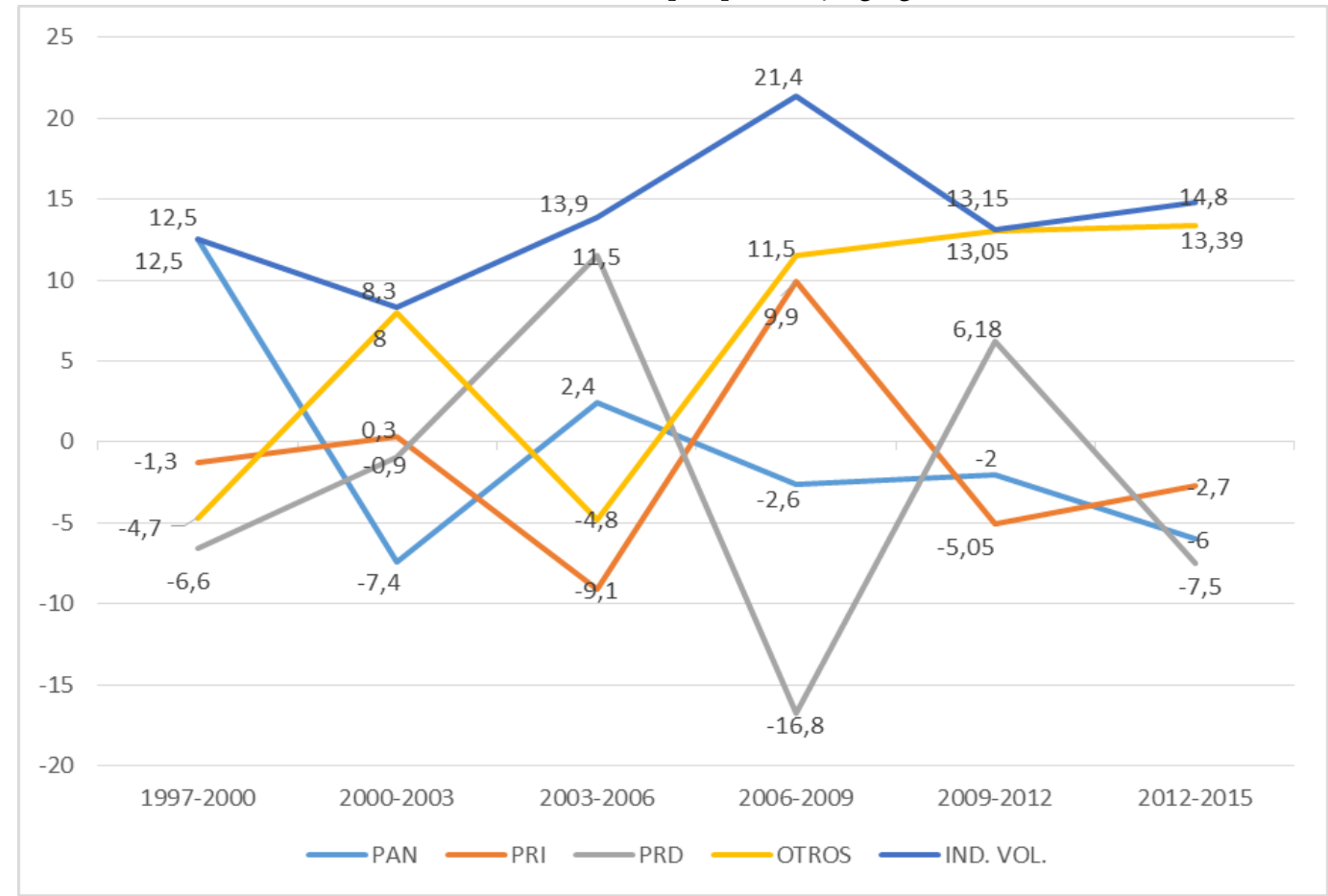

Fuente: Elaboración propia, datos IFE-Atlas electoral y Memoria electoral 2014-2015-INE.

Como se puede apreciar, en el Gráfico 2, el indice de volatilidad agregado es de 14,8, siendo el PRD el partido menos estable, zigzageando sus porcentajes entre una elección y otra, registrando signos positivos en elecciones presidenciales y negativos en las legislativas intermedias, con la sola excepción del periodo 1997-2000 cuando el comportamiento fue inverso, pues su porcentaje electoral fue superior en aquella que en esta en 7,4\%. Las causas de este comportamiento electoral errático del PRD se debe al impacto de los liderazgos carismáticos que han estado en el centro de cada elección y de sus niveles de popularidad. En 1997, el ingeniero Cuahtemoc Cárdenas obtuvo una contundente victoria electoral en la primera elección de autoridades del ejecutivo y del legislativo en el Distrito Federal, capital del país, que antes de esta, la capital se administraba políticamente por un regente o alcalde nombrado directamente por el Presidente de la República. Cárdenas logró sus más altos niveles de popularidad en ese año, después de las elecciones de 1988 cuando encabezó el Frente Democrático Nacional como candidato a la presidencia de la república, elección histórica que marca un parteaguas en la historia del régimen 
hegemónico en México. Su popularidad le alcanzó para ganar con amplio margen la Jefatura de Gobierno del Distrito Federal que en aquel año, concentraba casi el 11\% del padrón electoral nacional la amplia campaña mediatica derivada del acceso legal de los partidos a los medios masivos de comunicación (Radio y TV), que estableció como prerrogativa la reforma electoral de 1996 impactó la votación nacional del partido. Una intensa campaña de desprestigio desde el gobierno y las televisoras nacionales (Televisa y TV-azteca) mermaron su popularidad hacia el año 2000 cuando ocurrió la alternancia en la Presidencia de la república a través del PAN y su candidato Vicente Fox Quezada. Cárdenas apenas obtuvo el 16,64\% de los votos, $17 \%$ en las legislativas en el conjunto de los partidos de la alianza por México en el que además del PRD participaron aliados el Partido del Trabajo (PT), Convergencia (CPP), el Partido Alianza Social (PAS) y el Partido Sociedad Nacionalista (PSN), contra 25,70\% obtenido en las legislativas de 1997 lo cual explica su volatilidad de signo negativo en este periodo.

El segundo partido con las mayores fluctuaciones que impactan sus indices de volatilidad es el Partido Revolucionario Institucional (PRI), si bien las caídas de sus porcentajes son mínimos de los ciclos 1997-2000 y 2000-2003, es drástica de este ultimo con respecto a 2006, con una impresionante recuperación del signo positivo para las elecciones de 2009 para caer de nuevo en las elecciones de 2012 a pesar de su victoria electoral Presidencial, para seguir su tendencia a la baja en las elecciones de 2015. De los 6 ciclos electorales, el PRI registra baja en 5 y aumento solo en una, la de 2009. La volatilidad mostrada por este partido y su disminución porcentual a lo largo del periodo, explica también la fragmentación del sistema de partidos, porque las perdidas del PRI no tuvieron como destino los otros dos partidos principales PAN y PRD. Al contrario, estos también muestran alta volatilidad.

El PAN el otro partido principal del sistema, según se observa en el Gráfico 2, tiene alta volatilidad de signo positivo en el ciclo 1997-2000 y negativo en 20002003, y si bien recupera la tendencia positiva en el ciclo 2003-2006 por su triunfo en la elección presidencial del 2006, el margen de victoria estuvo lejos del obtenido por este partido en las elecciones del año 2000. Los últimos tres ciclos electorales la caída del PAN ha sido permanente, sus fluctuaciones en términos de volatilidad han sido menores, pero también sus porcentajes electorales, obteniendo en las elecciones de 2015 el peor resultado de todas las del periodo. Este mismo resultado se observa en los casos del PRI y del PRD, lo cual explica la caída en los niveles de concentración electoral analizados en el apartado anterior. 
Por el contrario, los "otros" partidos, que antes del inicio del proceso de desinstitucionalización del sistema plural tripartidista de partidos apenas sumaba en su conjunto menos de $10 \%$ del total de votos hasta el 2006, alcanzó la cifra de $18,32 \%$ en 2009 ; de $21,48 \%$ en 2012 y de $38,94 \%$ en las elecciones de 2015 . Las pérdidas acumuladas de los tres principales partidos significaron ganancias directas para los "otros partidos" lo cual refleja fielmente el indice de volatilidad de este rubro que tuvo signos negativos en los ciclos 1997-2000 y 2003-2006, pero impresionantemente positivos en los últimos tres ciclos: 2006-2009, 2009-2012 y 2012-2015, como se observa en el gráfico. Son muchas las variables que intervienen en estos resultados, el principal, la disminución del partidismo traducida en desafección hacia las tres fuerzas tradicionales, el descontento con los partidos de la oposición que hicieron en general malos gobiernos, pobre desempeño económico y peores resultados en los rubros de seguridad ciudadana, combate a la pobreza y la desigualdad, en general muy por debajo de las expectativas generadas por ellos mismos ante la ciudadanía en el prolongado proceso de transición a la democracia. En respuesta, la ciudadanía se alejó en porcentajes más o menos elevados del partidismo y disminuyó sensiblemente sus proporciones de participación electoral, por debajo del 50\% en las elecciones intermedias desde el año 2003 (MORALES GARZA et al., 2011), la otra variable relevante son las escisiones de grupos y líderes de los partidos tradicionales que han formado sus propios partidos de 2003 a la actualidad: el Partido Nueva Alianza (PANAL) se formó con las bases del Sindicato Nacional de Trabajadores de la Educación (SNTE) dirigido entonces por Elba Esther Gordillo Morales que antes de la escisión militaban en el PRI; el Partido Movimiento de Regeneración Nacional (MORENA) de Andrés Manuel López Obrador militaban hasta el 2012 en el PRD y destacadas personalidades del PRI, PAN y PRD son tránsfugas hacia "otros" partidos o " actores independientes" como los casos de Enrique Alfaro en Jalisco (del PRI a Movimiento Ciudadano), Jaime Rodriguez "el Bronco" (del PRI a Independiente), Manuel Clouthier (del PAN a Independiente), Cuahtemoc Cárdenas, Alejandro Encinas entre otros, del PRD a Independientes.

Finalmente de volatilidad agregada ha mantenido en general una tendencia a la alza, de 8,3 registrada en el ciclo 2000-2003, a 14,8 en el ciclo 2012-2015, con un pico excepcional en el ciclo 2006-2009 de 21,4 para promediar 14,0 en el periodo que se analiza, lo que refleja muy bien las tendencias de zigzag de los tres partidos tradicionales con signos negativos en las variaciones en cada ciclo salvo excepciones y por el contrario signos positivos en las variaciones de los "otros partidos". La 
24 | José Manuel Luque Rojas

tendencia de desafección hacia los partidos tradicionales se observa con mucha nitidez a través de esta variable de volatilidad.

\section{Número Efectivo de Partidos}

El numero efectivo de partidos (NEP) no sólo es indicador de aumento de la pluralidad electoral en un régimen democratizado, si no también y de manera principal es útil para medir el aumento o disminución del número de partidos relevantes en un sistema determinado. En este caso, aunque no necesariamente ocurre en otros escenarios, el indice NEP se ve afectados por las mismas variables políticas que la volatilidad: la desafección y el desencanto ciudadano hacia los partidos tradicionales del sistema. El Gráfico 3 muestra el proceso de modificación del número de partidos relevantes el cual si bien ha sido constante desde 1994, existen al igual que en el indice de volatilidad picos en los ciclos electorales 2003-2006 y 20122015. 
Gráfico 3 - Evolución del Indice NEP 1994-2015, elecciones de diputados de RP

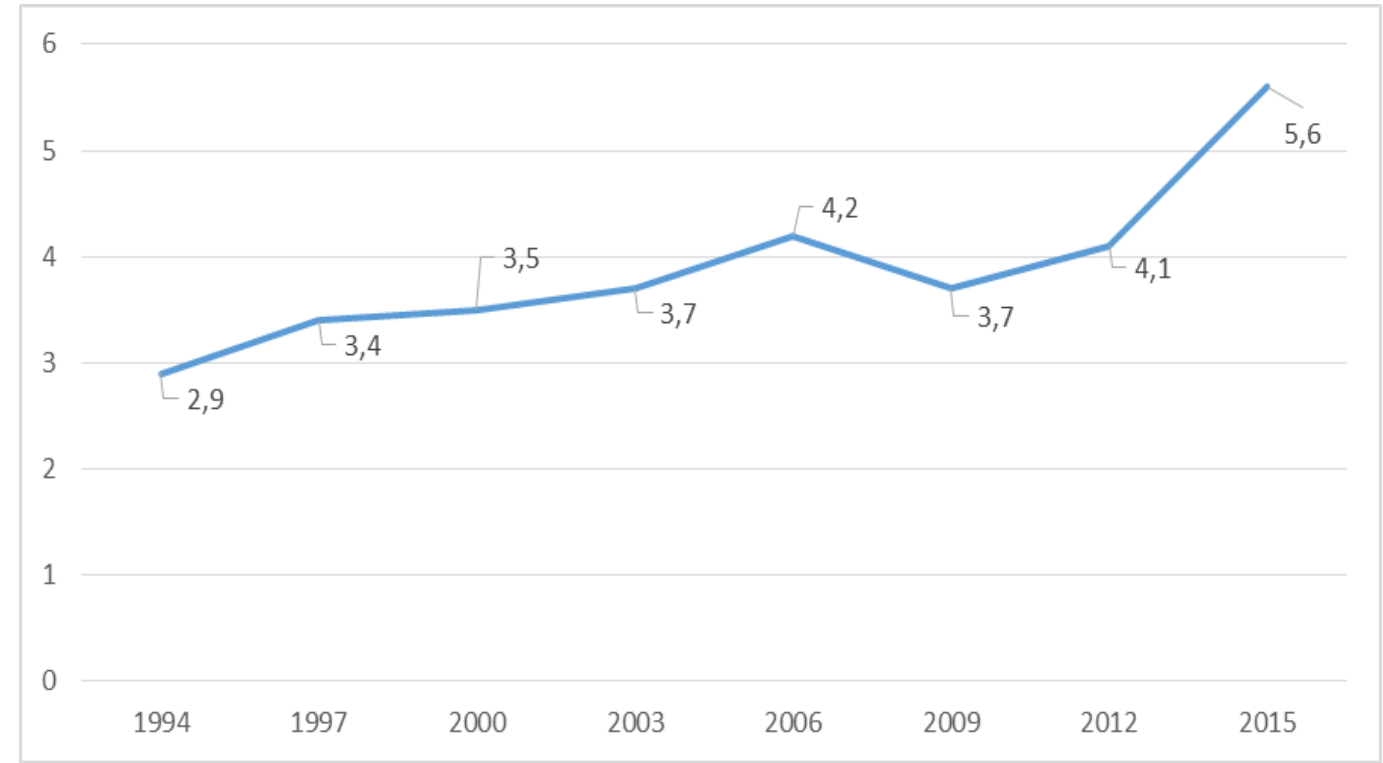

Fuente: Elaboración propia, datos del INE-Atlas electoral.

Cuáles son las variables que explican en este caso el aumento del número efectivo de partidos? La respuesta obvia, es el constante incremento de la desafección hacia los tres principales partidos políticos que se expresan no solo en los niveles de concentración electoral y de volatilidad si no en la emergencia de nuevos actores políticos agrupados en partidos antes marginales y a partir de 2015 en las "candidaturas independientes" que configuran en su conjunto un nuevo polo de ciudadanos no conformes con el desempeño de los gobiernos y la representación de los partidos tradicionales al menos en el ámbito federal, aunque también es observable la misma tendencia en los ámbitos de los estados y municipios. En el Gráfico 4 se observa la caída más o menos constante del desempeño de los partidos políticos tradicionales y como el elector inconforme se agrega cada vez en mayores proporciones en la categoría "otros" que agrupa como ya se dijo a partidos antes marginales o de nueva creación, candidaturas independientes o el voto en blanco o nulo. 
Gráfico 4 - Tendencia rendimiento electoral de los partidos1994-2015

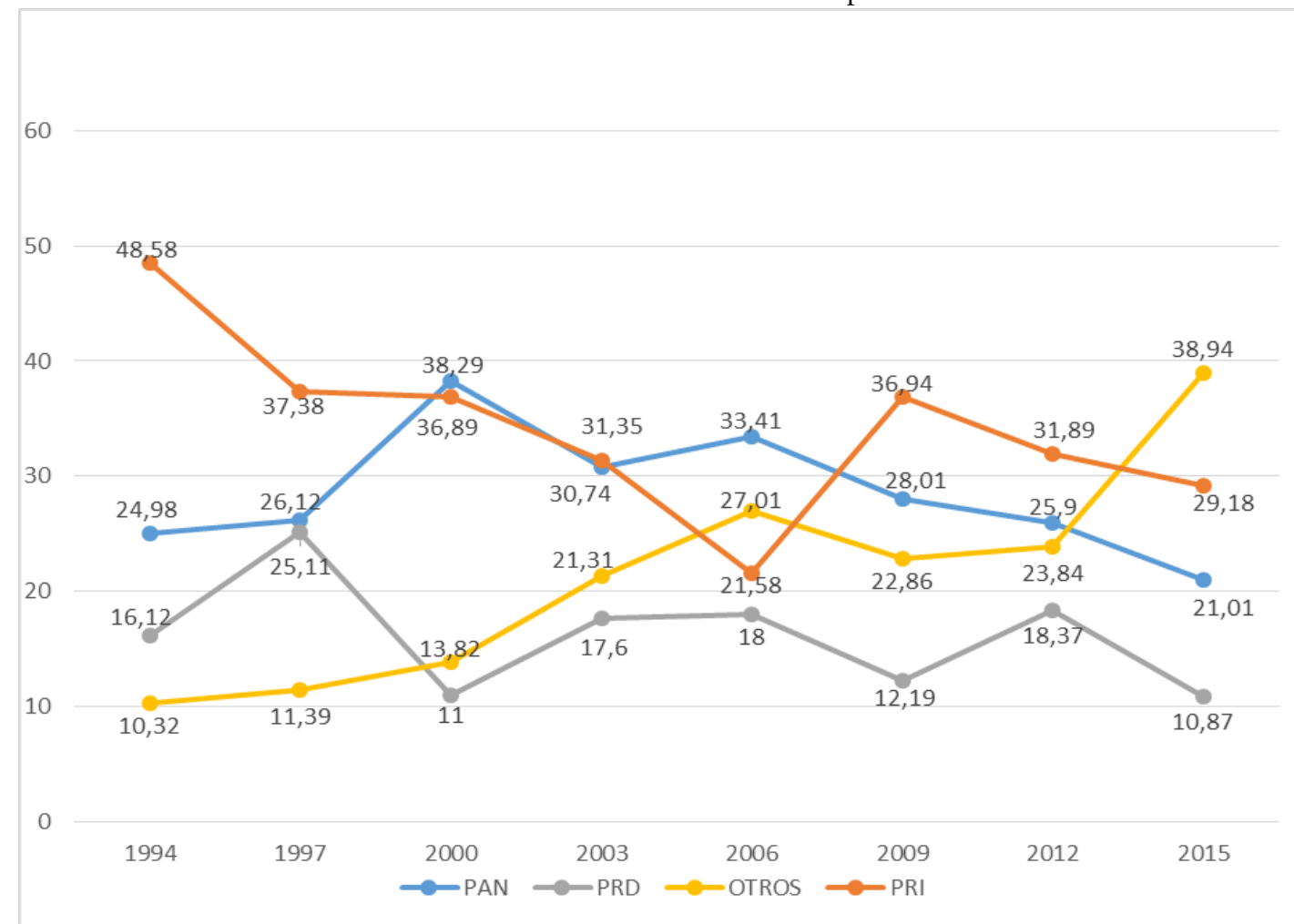

Fuente: Elaboración propia, datos del IFE-INE, Atlas electoral 1991-2012 y memoria 2015.

Desagregando los datos del gráfico el PRI perdió casi 20 puntos porcentuales de apoyo electoral entre 1994 y 2015 pasando de 48,58\% a 29,18\%, el PAN pierde $17,28 \%$ al pasar de $38,29 \%$ en el ańo 2000 a $21,01 \%$ en el 2015 , mientras el PRD pierde 14,24 puntos porcentuales de apoyo al registrar 25,11\% en 1997 y apenas $10,87 \%$ en el 2015 . Son estas las cifras del desencanto ciudadano hacia los partidos que protagonizaron la transición desde la hegemonía priista a la democracia electoral: el PRI y la oposición.

\section{Algunas causas que ayudan a explicar la crisis del sistema de partidos tradicional}

Las causas de la desafección son múltiples y variadas, pero son evidentes las promesas de cambio no cumplidas al llegar la oposición al gobierno, las transformaciones institucionales no se concretaron por falta de voluntad, pero 
también porque la de 2000 y 2006 fue una transición a medias. El PAN ganó las presidencias de 2000 y 2006 pero el PRI y sus aliados ganaron el Congreso, el PRD congruente con sus principios ideológicos no apoyo las reformas en materia económica propuestas por el PAN y éste no propuso cambios en las estructuras institucionales del Estado y el régimen político. En la práctica las instituciones creadas por el PRI durante la hegemonía siguieron vigentes en los 12 años de gobiernos panistas. Lo cual provocó desde enojo y disgusto hasta desinterés en la política por parte del ciudadano: todos son iguales dicen.

\section{Pacto por México}

La estrategia del PRI de bloquear los escasos cambios propuestos en materia económica por los gobiernos del PAN resultó exitosa. Regresó al gobierno en el 2012 con el triunfo de Enrique Peńa Nieto (EPN). Éste, impulsó los cambios que en teoría debieron haber impulsado los gobiernos de transición pero que su escasa iniciativa política y su (in)capacidad de gestión y negociación no le permitieron. Peña promovió a iniciativa del PRD el "Pacto por México" que a la postre derivó en la concreción, no sin problemas, de las llamadas reformas estructurales o modernizadoras de México y giraron en torno a cinco acuerdos básicos:

1. Sociedad de Derechos y Libertades;

2. Crecimiento Económico, Empleo y Competitividad;

3. Seguridad y Justicia;

4. Transparencia, Rendición de Cuentas y Combate a la Corrupción, y;

5. Gobernabilidad Democrática.

Que se tradujeron en la creación de decenas de reformas constitucionales y de leyes nuevas o actualizadas que derrumbaron viejos esquemas de operación en materia económica, política social, justicia, gobierno, corrupción y derechos humanos principalmente. Las reformas más importantes para el presente y el futuro para bien o para mal son: en materia laboral, educativa. Financiera, hacendaria, energética, telecomunicaciones, del sistema y el regimen político, ampliación de derechos civiles y políticos, sistema de justicia penal, y el sistema nacional anticorrupción. Los cambios llevados a cabo, no obstante, tuvieron el efecto contrario en la ciudadanía, la profundización de la crisis de credibilidad que ya arrastraban los partidos tradicionales desde 2006 se agrava en el 2015. Las causas del efecto adverso de las reformas son muchas y merecen estudios por separado y de conjunto imposible de 
exponer en esta colaboración, sin embargo menciono algunas que son observables a simple vista.

Las transformaciones institucionales llegaron al regreso del PRI al gobierno federal, después de haber sido expulsado en el año 2000. El pacto por México concretó cambios en las instituciones del estado y el régimen y otros aspectos de gran envergadura. Los cambios son demasiado recientes para observar mejoras sustanciales en la vida social, económica y política de los mexicanos y en cambio si ha tenido efectos negativos en sectores concretos que se distinguen por su alta capacidad de lucha y protesta: los maestros, la iglesia y los magnates de sectores estratégicos como las telecomunicaciones del sector empresarial, además, no todas las reformas son pertinentes, también algunas de ellas han mostrado producir efectos adversos a los esperados al menos en el corto plazo. Algunas de ellas, empero, han provocado fuertes reacciones de diversos sectores sociales y políticos al sentirse, con razón o sin ella, afectados en sus derechos presentes y no ven más que perjuicios en el futuro, es el caso de la reforma educativa que ha puesto en pie de guerra tanto a la disidencia como al propio sindicato nacional de maestros: la Coordinadora Nacional de Trabajadores de la Educación (CNTE) y el Sindicato Nacional de Trabajadores de la Educación (SNTE) respectivamente, el primero tradicionalmente ligado a la izquierda y el segundo al oficialismo desde el PRI y una parte de este desde el partido Nueva Alianza (PANAL). Pero también se pusieron en pie de lucha los partidos contra sus propias reformas; el PRD contra la energética al considerar que despoja a los mexicanos de su patrimonio (Petróleo, gas natural y energía eléctrica), quien a la firma del Pacto abogaba por el mantenimiento del monopolio estatal en la explotación de estos recursos, sus propuestas de reformas para este sector no incluía la apertura a las inversiones de capital privado, la propuesta de Acción Nacional y del PRI se impuso y se acordó una apertura casi total del ramo en todos sus procesos; mientras que el PAN se declaró abiertamente en contra de la reforma hacendaria, paradogicamente todas estos cambios fueron acordadas en el Pacto por México en el que estaban incluidas las tres fuerzas políticas principales ahora en decadencia.

Las reacciones fuera del PRI, PAN y PRD contra el conjunto de las reformas estructurales han sido particularmente potentes por parte de sectores muy poderosos del sector empresarial, especialmente contra la reforma en telecomunicaciones en el que encontraron ocasión de enfrentarse los gigantes de la telefonía fija y celular (Telmex-Telcel) contra el duopolio de la televisión abierta y de paga, (Televisa y TVAzteca), ambas queriendo invadir el giro de los otros. El planteamiento inicial de la reforma pretendía abrir ambos giros del sector a nuevos empresarios y los dos 
presionaron por separado a las bancadas del PRI y del PAN que terminaron modificando la propuesta original y cerrando la puerta a la competencia externa y se integraron mutuamente en los giros del otro por parte de los dos grupos, en la práctica la reforma que pretendía acabar con los monopolios del sector terminó reforzándolos, lo que hizo a la fracción parlamentaria del PRD votar en contra de ésta en particular a pesar del acuerdo inicial de apoyarla, también votó en contra de la energética por definiciones ideológicas, mientras que el PAN por razones más pragmáticas voto en contra de la hacendaria en todos los casos la bancada del PRI contó con el apoyo de uno de los dos partidos, lo que permitieron sacar adelante el conjunto de los cambios pactados.

Las otras fuerzas que se opusieron a las reformas en general además de la disidencia magisterial (CNTE) contra la educativa y el resto de las reformas son diversos sectores sociales y políticos esencialmente conservadores. La iglesia católica y otras religiones han expresado rechazo total y fuertes expresiones de protesta bien organizada contra las iniciativas presidenciales que protegen a minorías de la discriminación a la que han sido sometidos históricamente, son los casos de la iniciativa de matrimonio igualitario, la ley de niñas, niños y adolescentes y la legalización de la marihuana, la primera y la ultima no pasaron.

Como puede verse son muchos los sectores y actores sociales y políticos que por alguna u otra consideración con razón o sin ella han visto en las "reformas estructurales" materializado el demonio que representan las fuerzas políticas tradicionales del régimen y han abandonado sus trincheras para sumarse a la disidencia que ha fortalecido otras opciones políticas. Otra variable de suma importancia en esta perspectiva es el fortalecimiento del populismo que parece aglutinar en torno a sí el descontento de unos y otros.

\section{MORENA y Andrés Manuel López Obrador}

Pudiera pensarse que las causas de fondo del rechazo a los partidos tradicionales tiene que ver también con la distancia entre la retórica partidista que luego no encuentra sustento en las acciones de gobierno de estos partidos tanto en el ámbito federal como de los estados y municipios. Si esa retórica sin contenido fuera la causa, entonces la ciudadanía sería renuente a discursos de líderes de cualquier partido, pero no es así, el discurso de uno de los líderes de un partido político el Movimiento de Regeneración Nacional (MORENA) promete justamente acabar con toda la clase política corrupta, generar empleos bien pagados para todos, encarcelar a 
políticos y empresarios mafiosos y cambiar el modelo económico a uno más domestico, sustraer a México de las fauces del capitalismo voraz que consume los recursos y la riqueza nacional. Como puede verse, es éste, en esencia un discurso populista, muy parecido, casi idéntico al de los presidentes en funciones de Venezuela, de Bolivia, de Ecuador y Nicaragua. Este discurso ha tenido una excelente recepción por parte de la ciudadanía disidente, las encuestas colocan de nuevo a Andrés Manuel Lopez Obrador en la cresta de la ola de la sucesión presidencial en el 2018. Es casi seguro que las alternativas a la crisis que vive México que propone AMLO fracasen, no tienen sustento la mayoría de ellas en el contexto global y de las capacidades internas del país, pero es altamente probable que sea el ganador de las elecciones presidenciales que se avecinan, o al menos estará como en el 2006 y 2012, en la antesala de lograrlo.

\section{La corrupción institucionalizada}

Parte del problema de México hoy, como lo afirma el propio AMLO, es la corrupción institucionalizada que se observa cada vez con más frecuencia en los escándalos protagonizados por la elite gubernamental emanada de los partidos políticos tradicionales, pero también de los "otros partidos", los casos documentados de la "Casa Blanca” del Presidente Peña y de su esposa, las casas de Malinalco, Estado de México del Ex secretario de Hacienda y las innumerables propiedades del Secretario de Gobernación de EPN, son junto con las acciones de corrupción y fraude de exgobernadores evidencia que fortalece el discurso restaurador que alimenta como un moustro la opción populista de AMLO.

Aunque vale decir, que hablando de corrupción y escándalos nadie de la clase política se escapa, incluido el propio AMLO, quien inaugura desde su paso por la Jefatura del Gobierno del Distrito Federal (hoy ciudad de México) (2000-2006) los escándalos de corrupción. El caso de los video-escándalos del profesor René Bejarano, (Secretario Particular y operador político de AMLO), Carlos Imaz (Delegado en Tlalpan) y Gustavo Ponce (Secretario de Finanzas del Gobierno del Distrito Federal, en el periodo de AMLO) lo salpicaron fuertemente y de paso también involucraron a la dirigencia nacional del Partido de la Revolución Democrática (PRD) y sus liderazgos: Rosario Robles (Presidenta del Comité Ejecutivo Nacional), entre muchos otros distinguidos líderes políticos y altos funcionarios de gobiernos de la izquierda (MARTÍNEZ GONZÁLEZ, 2005).

Por su parte, los probados y documentados actos de corrupción de connotados líderes del gobernante PAN y del PRI como bien lo documentan Lorenzo 
Córdova y Ciro Murayama, respecto del uso y abuso del dinero en las campañas presidenciales y legislativas del ańo 2000: los casos de "Amigos de Fox" y el PEMEXGATE (CÓRDOVA y MURAYAMA, 2006) que salpicaron también al PRI y al PAN y los sucesivos excesos documentados por la periodista Anabel Hernández del desempeño del gobierno del cambio de Vicente Fox, en los que describe: tráfico de influencias, desvío y mal uso de recursos públicos y diversas violaciones a la ley por parte de los hijos de Martha Sahagún de Fox que manchó el primer gobierno de alternancia en México (HERNÁNDEZ, 2005 y 2007).

En este mismo sentido, Hernández publica en el 2008 otro libro en el que documenta actos de corrupción en los que habrían incurrido el ex presidente Felipe Calderón Hinojosa y sus cómplices como los denomina ella en el titulo de su obra, en él da cuenta de los actos de debilitamiento del Instituto Federal Electoral a partir del nombramiento de Luis Carlos Ugalde como presidente del Consejo General, no por parte de Ugalde, por cierto, si no de parte de altos funcionarios de la Junta General del IFE y del Registro Federal de Electores, que fueron el sustento de los actos que desembocaron en la elección poco transparente de julio de 2006 en la que ganó Felipe Calderón, hasta la celebración de contratos de obra del Gobierno Federal a connotados empresarios donantes ilegales de la campaña calderonista según la autora de referencia (HERNANDEZ, 2008).

La cadena de actos de corrupción en las más altas esferas del poder político en México ha sido particularmente grave en el actual sexenio del priista Enrique Peña Nieto. Tras su relativamente holgada victoria sobre el ex perredista Andrés Manuel López Obrador en el 2012, el señalamiento de este sobre acciones ilegales de su campaña por el caso Monex (RAMÍREZ CUEVAS, 2012; ORDAZ BAUTISTA, 2014) inició un gobierno con amplia legitimidad social y política, fortalecida en parte por la firma del Pacto por México entre su gobierno y los líderes de los tres principales partidos políticos del país: PRI, PAN y PRD. El pacto se tradujo en cambios profundos en la estructura institucional y económica del Estado mexicano, las llamadas reformas estructurales que se plantearon como necesarias desde el inicio del gobierno de Ernesto Zedillo (1994-2000) y que debido a los resultados electorales para la integración del poder legislativo que ha arrojado gobiernos divididos desde entonces, no había sido posible concretarlas ni en la segunda parte del gobierno zedillista, ni en los de Vicente Fox (2000-2006) y de Felipe Calderón (2006-2012), las principales reformas fueron: la laboral, la hacendaria, financiera, telecomunicaciones, energética, la política y educativa (BARRIENTOS DEL 
MONTE y AÑORVE AÑORVE, 2014). Estos cambios en los primeros dos años del sexenio le otorgó al gobierno un bono adicional de legitimidad, que fue derrochado meses después de concluidas las reformas, con los escandalosos casos de "Ayotzinapa" (MALDONADO, 2015) y la "Casa blanca" (LIZÁRRAGA et al., 2015).

Ambos casos de corrupción y otros que se sumaron en los meses siguientes por parte de miembros del gabinete del Presidente Peńa como el de las casas de "Malinalco" de Luis Videgaray (Secretario de Hacienda) y las propiedades de Miguel Ángel Osorio Chong, (Secretario de Gobernación). El gobierno intentó dar explicaciones que sólo sirvieron para profundizar la desconfianza y sumar actos de corrupción, como el encargo hecho por el propio Presidente Peña a un subordinado del gabinete, el Secretario de la Función Pública, Virgilio Andrade, que hiciera una investigación del "affaire de la Casa Blanca" y de las propiedades del resto de los altos funcionarios de su gobierno, meses después, Andrade rindió su informe en la que en relación con los casos de la compra de la casa en Ixtapan (de Peña); la de Malinalco (de Videgaray); y la de Lomas de Chapultepec (de Angélica Rivera, esposa del Presidente), así como en contratos otorgados a Grupo Higa y a Constructora Ixtapan, concluye que, "en ninguno de ellos, existe conflicto de interés" (MARTINEZ HUERTA, 2015).

El informe no dejó satisfecho a nadie y la popularidad del gobierno siguió en caída libre pasando de 52\% de aprobación de su gestión en febrero de 2014, a 40\% en noviembre del mismo año tras los hechos de Ayotzinapa y los escándalos de corrupción, a 30\% para abril de 2015, según las media de diversas casas encuestadoras (VILLAMIL, 2016) y recientemente la ultima encuesta publicada por el periodico el universal el 20 de noviembre de 2016, desciende a 25\% de aprobación el nivel más bajo para un presidente mexicano de las últimas décadas ${ }^{1}$.

Estos actos de corrupción, conjugado con un entorno internacional desfavorable en materia económica y escasos o nulos resultados en materia de combate a la pobreza y el aumento de la violencia generada por el crimen organizado, hacen que la percepción casi generalizada de la población califique el desempeño del gobierno de EPN en materia de seguridad totalmente ineficiente, reforzado con el reporte oficial del INEGI y otras fuentes no gubernamentales de que los índices delincuenciales se han mantenido en los mismos niveles o se han elevado, después de los picos generados durante la gestión de la crisis de seguridad iniciada en el gobierno del Presidente Felipe Calderón. En suma, los saldos negativos de las reformas del

\footnotetext{
${ }^{1}$ Disponible <amp.eluniversal.com.mx>. Acceso en 20 nov. 2016.
} 
Pacto por México, el fracaso en las políticas publicas de combate a la pobreza y al crimen organizado además de los escándalos de corrupción del gobierno federal y de los estados y municipios de los tres partidos (Guerrero y San Luis Potosí y Morelos del PRD; Sonora y Sinaloa del PAN; Veracruz, Chihuahua, Quintana Roo, Coahuila y Durango del PRI le han pegado de manera particular al gobierno de EPN, pero también a los partidos políticos tradicionales de la oposición.

Derivado del reconocimiento de que el principal problema del aumento del apartidismo y el fortalecimiento de las opciones populistas, la clase política mexicana agrupada en los partidos tradicionales incluyó entre las reformas del pacto por México cambios en las leyes para castigar actos de corrupción y mejorar los procesos de rendición de cuentas y transparencia. También quizá porque los reportes de los diversos organismos nacionales e internacionales en los últimos años sobre "estado de derecho en México" han señalado el problema de la corrupción como una de las grandes debilidades de la democracia mexicana, por ello, en el año 2015 el gobierno de la república y los partidos, a través del "Pacto por México" aprobaron la reforma constitucional para crear el llamado sistema nacional anticorrupción que incluye cambios en al menos 3 leyes federales y la creación de 4 nuevas, así como, de un conjunto de instituciones encaminadas a administrar los mecanismos y procedimientos nuevos para combatir la corrupción institucionalizada del país. Las leyes a crear y/o reformar son las siguientes: nuevas: 1. Ley General del Sistema Nacional Anticorrupción; 2. Ley General de Responsabilidades Administrativas; 3. Ley Orgánica del Tribunal Federal de Justicia Administrativa y; 4. Ley de Fiscalización y Rendición de Cuentas de la Federación. En tanto que las reformadas: 5. la Ley Orgánica de la Procuraduría General de la República; 6. el Código Penal Federal, y; 7. la Ley Orgánica de la Administración Pública Federal.

El problema es que el sistema es de reciente aprobación y por principio general, no se aplica a actos pasados de corrupción y las existentes son benévolas y con inmensas lagunas para evadir responsabilidades, el ejemplo más claro son los de los casos de exgobernadores de Tamaulipas, y el de Veracruz, que están prófugos de la justicia y otros que están bajo proceso judicial o en etapa de investigación para generar acusaciones que prácticamente están exentos de la aplicación de las nuevas leyes del sistema. 
34 | José Manuel Luque Rojas

\section{Resultados y conclusiones: La crisis del sistema de partidos y la coyuntura electoral de 2018}

En general las expuestas en el apartado anterior son las causas evidentes de la desafección ciudadana hacia los partidos tradicionales. Las elecciones presidenciales son en junio de 2018. Las encuestas marcan desde hoy a AMLO el actual presidente del partido MORENA como el rival a vencer en la contienda. A 11 meses de que inicie el proceso electoral la tendencia de desafección hacia los partidos tradicionales le pinta bien al lider populista de la izquierda y mal a la clase política y los partidos tradicionales. El Gráfico 5 muestra los datos.

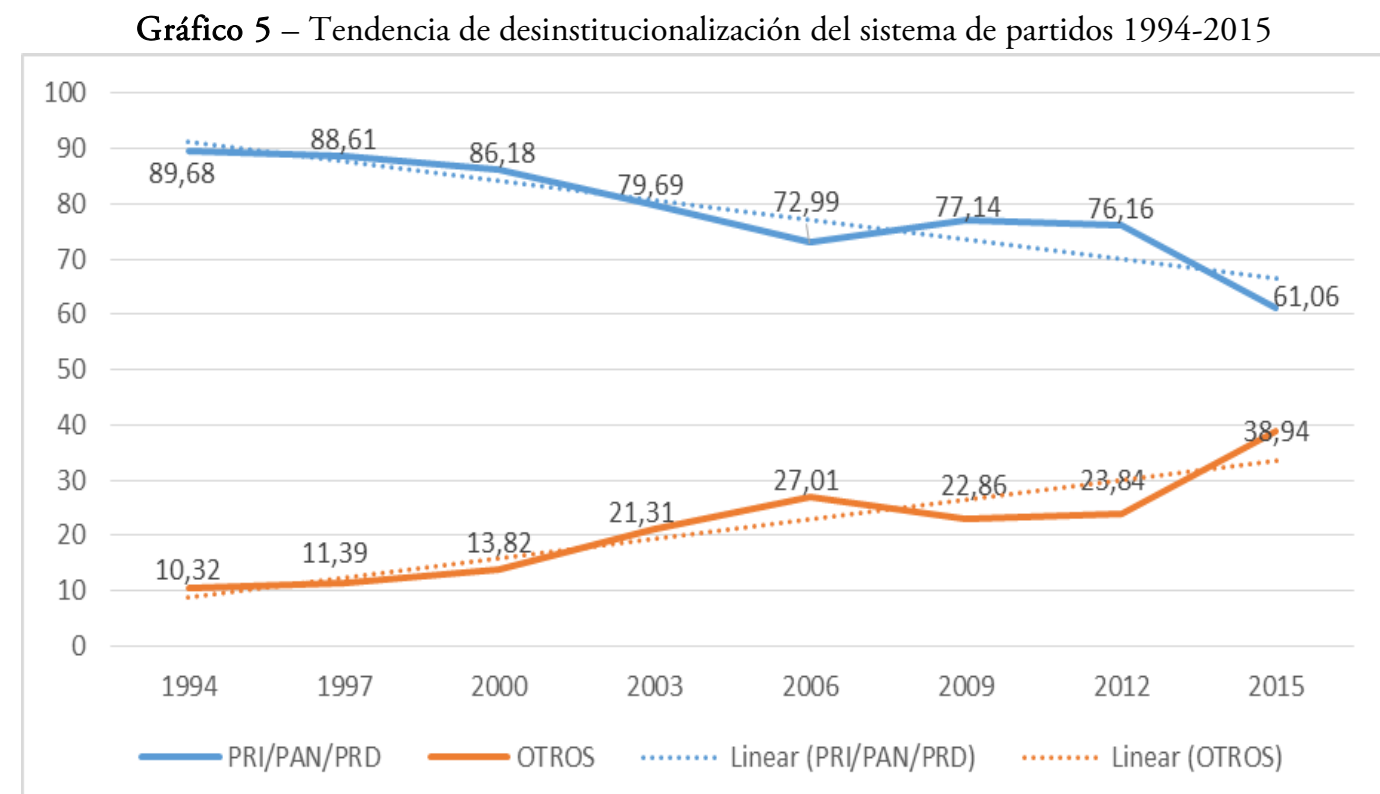

Fuente: Elaboración propia, datos del IFE-atlas electoral 1991-2012 y memoria electoral 2014-2015 del INE.

Hasta el año 2012, AMLO jugó electoralmente del lado del establishment politico, fue candidato presidencial en 2006 y 2012 por el PRD, para las elecciones intermedias de 2015 fundó su propio partido (MORENA) y le ha declarado la guerra a los partidos tradicionales, esto explica en parte, el crecimiento de la oposición al sistema de partidos y acelera su desinstitucionalización, es decir, introduce incertidumbre en los resultados de las elecciones y del ejercicio de la política y el gobierno a partir de pasada la coyuntura electoral de 2018, cualquiera que sea el resultado pero especialmente si resulta ganador. 
Las probabilidades de que el sistema de partidos tradicional sea derrotado en esa coyuntura es alta, la franja de votantes que pertenecieron durante décadas al PRD ahora han migrado según resultados de las elecciones locales de 2016 mayoritariamente a MORENA y se espera que el realineamiento electoral se profundice en el 2018, si esto es así, y las encuestas resultan ratificadas en el proceso electoral, se estará inaugurando un nuevo sistema de partidos con la expulsión de actores principalmente de la izquierda del escenario central y la disminución de la influencia de los otros dos. Las opciones populistas, lo muestran varios casos de américa latina en la actualidad tienden a mantenerse en el poder valiendose del gobierno y las instituciones del estado, aun con mayor fuerza que lo hacen los partidos más comprometidos con los métodos democráticos. Aunque no son infalibles, las derrotas electorales del peronismo en Argentina, la caída de Dilma Roussef en Brasil y la crisis del régimen de Nicolás Maduro en Venezuela, muestran que pueden durar tiempo en el poder, pero como les pasa ahora a los partidos tradicionales mexicanos, también pierden credibilidad y elecciones.

México no es inmune al populismo que ha esquivado hasta el día de hoy, pero los partidos tradicionales han hecho lo necesario para perder la centralidad, hacia allá se encamina la democracia mexicana, son muchas las causas que han llevado a esta situación al régimen mexicano, en esta colaboración apenas esbozamos unas cuantas, pero la desafección afecta a los tres partidos en todas las regiones del país, a pesar de que salvo el PRI, los otros dos son partidos regionales con fuerte presencia en algunas y nula en otras, Son corruptos, y sus procedimientos democráticos internos dejan mucho que desear, nada que envidiar al caso Argentino que el politologo de ese país Martin D'Alessandro (2013) cuando dice “tenemos un sistema posbipartidista, territorializado, poco institucionalizado, poco ideológico, impredecible y con dificultades para producir liderazgos o generar cambios" ${ }^{2}$.

La caída de los sistemas de partidos nunca son buenas noticias para las democracias, hay decenas de ejemplos de ello, desde la decadencia del sistema partidista de la república de Weimar que le abrió el paso al Nazismo en Alemania, hasta el derrumbe del sistema partidista en el Perú a finales de la década de 1990 que llevó al triunfo a Alberto Fujimori con las consecuencias ya conocidas para la democracia. Los partidos deberán reequilibrarse con los mismos o nuevos actores que expresen sin ambages el compromiso con las instituciones y los procesos

\footnotetext{
${ }^{2}$ Disponible <http://elestadista.com.ar/?p=3462>. Acceso en 20 nov. 2016.
} 
democráticos, deberán como dice Javier Duque "adaptarse o transformarse" (DUQUE, 2016). Eso sería lo deseable para no desandar lo ya andado ni seguir los pasos de nuestros hermanos de América Latina que han pasado por regímenes que solo garantizan polarización y pobreza.

- José Manuel Luque Rojas é Doutor em Estudos Sociais, pela Universidade Autônoma Metropolitana. É Professor Investigador da Universidade Autônoma de Sinaloa. E-mail: luque-m@hotmail.com.

\section{Referências}

AGUILAR, Héctor; MEYER, Lorenzo. A la sombra de la revolución mexicana, México, Cal y Arena. 1991.

ALCÁNTARA SAEZ, Manuel. Partidos políticos en America Latina: precisiones conceptuales. In: PNUD. La democracia en América Latina, hacia una democracia de ciudadanas y ciudadanos, contribuciones para el debate. Buenos Aires: Aguilar, Altea, Taurus, Alfaguara, 2004. p. 99-122.

BARRIENTOS DEL MONTE, Fernando; AÑORVE AÑORVE, Daniel. México 2013: acuerdos, reformas y descontento. Revista Ciencia Política. v. 34, n. 1, 2014. p. 221-247.

COLOMER, Joseph. Instituciones Políticas, Madrid, Ariel, 2001.

CÓRDOVA, Lorenzo; MURAYAMA, Ciro. Elecciones, dinero y corrupción. Pemexgate y Amigos de Fox, Mexico, Cal y Arena, 2006.

DE VRIES, Walter; TARRANCE, V. Lance Jr. The Tiket-Splitter a new forcé in american Politics. Eerdmans, Grand Rapids, 1972.

DUQUE, Javier. Los partidos políticos contemporáneos ¿Crisis, adaptación o transformaciones? Revista Folios, n. 31, octubre/2016.

PALLARÉS, Francesc y KEATING, Michael. Multilevel electoral competition: sub-state elections and pary systems in Spain. In. HOUGH, Dan; JEFFERY, Charlie (org.) Devolution and Electoral Politics. Manchester Manchester University Press, 2006. p. 96-118.

FIORINA, Morris.P. Divided goverment, New York: Macmillan, 1992.

HERNÁNDEZ, Anabel. La Familia Presidencial: El Gobierno del Cambio Bajo Sospecha de Corrupción, México, Grijalbo. 2005.

HERNÁNDEZ, Anabel. Fin De Fiesta En Los Pinos, México, Grijalbo. 2007.

HERNÁNDEZ, Anabel. Los Cómplices del Presidente, México, Grijalbo. 2008.

IFE-INE, Atlas electoral 1991-2012 y memoria. 2015.

JACOBSON, Gary Charles. The electoral origins of divided goverment competition in U.S. House election, 1946-1988. Boulder, Wetsviear Press. 1990. 
JIMÉNEZ, Margarita; SOLANO, Gabino. Procesos electorales en la república Mexicana (1980-2009). Instituto Internacional de Estudios Políticos Avanzados Ignacio Manuel Altamirano Universidad Autónoma de Guerrero. 2009.

LAAKSO, Markku; TAAGEPERA, Rein, Effective number of parties: A measure with application to West Europe. Comparative Political Studies, v. 12, n. 1, 1979. p. 3-27.

LIZÁRRAGA, Daniel; CABRERA, Rafael; HUERTA, Irving; BARRAGÁN, Sebastián. La casa blanca de Peña Nieto: La historia que cimbró un gobierno. México, Grijalbo. 2015.

MALDONADO, Tryno. Ayotzinapa. El rostro de los desaparecidos. México, Planeta. 2015

D'ALESSANDRO, Martin. El sistema de partidos. Revista el Estadista, n. 159, 2013 Disponible em $<$ http://elestadista.com.ar/?p=3462>. Acceso 2 dez. 2016.

MARTÍNEZ GONZÁLEZ, Víctor Hugo. Fisiones y fusiones, divorcios y reconciliaciones. La Dirigencia del PRD 1989-2004. México, Plaza y Valdés. 2005.

MÉXICO. Ley Federal de Organizaciones Políticas y procesos Electorales 1977. 1977.

MONTERO, José Ramón. Voto nacional y voto autonómico, la escisión del voto en las elecciones de 1986 en Andalucía. Revista Española de Ciencia Política, n. 42, 1988. p. 177-194.

MORALES GARZA, Martha; MILLÁN VALENZUELA, Henio; ÁVILA EGGLETON, Marcela; FERNÁNDEZ GARCÍA, Luis Alberto. Participación y abstencionismo electoral en México, CONACyT-IFE-UAQ. 2011.2 Disponible em <http://www.ceddi.uan.mx/webderecho/descargas/productividad/derechoconstitucional/RojaR\%20F igueroa\%20Edgar\%20Hugo.pdf>. Acceso 2 dez. 2016.

ONATE, Pablo; OCANA, Francisco. Las elecciones autonómicas de 1999 y las españas electorales, España, Reis. 1999.

ORDAZ BAUTISTA, Luis Fernando. El modelo de fiscalización de los partidos políticos. In. CEDE. El Nuevo Sistema Político Electoral Mexicano en 2015. México, CEDE. 2014.

PEDERSEN, Mogens. The dynamics of West European party system changing patterns of electoral volatility. European Journal of Political Research, v. 7, 1979. p. 1-26.

RAMÍREZ CUEVAS, Jesús. La presidencia comprada, México, endebate. 2012.

FLORES ANDRADE, Anselmo. Pasado y presente de los partidos nuevos y las asociaciones políticas nacionales en el sistema electoral mexicano. Cuestiones Constitucionales Revista Mexicana de Derecho Constitucional, [S.1.], jan. 2005. Disponible en <https://revistas.juridicas.unam.mx/index.php/cuestionesconstitucionales/article/view/5729/7517>. Acceso 09 dec. 2016

SANZ CAZORLA, Alberto, La escisión del voto en el sistema de gobierno multinivel espańol: ¿electores, candidatos, gobiernos o instituciones?, elecciones nacionales y autonómicas en Andalucía 1986-2004. In. Working Papers, Madrid, Universidad Autonoma de Madrid. 2005.

SARTORI, Giovanni. Partidos y sistemas de partidos, Madrid, Alianza editorial. 1987.

VILLAMIL, Jenaro; ESQUIVEL, Jesus. Entretelones de la visita ignominiosa de Trump. Revista Proceso, Edición 2079, 2016. 
38 | José Manuel Luque Rojas

\section{Documentos}

El Pacto por México. Disponible en

<http://www.foroconsultivo.org.mx/documentos/politicas_publicas/pacto_por_mexico.pdf>.

Accesso 2 dez. 2016.

Texto recebido em 04 de novembro de 2016.

Aprovado em 06 de dezembro de 2016. 